\title{
MINING MULTIDIMENSIONAL FUZZY ASSOCIATION RULES FROM A DATABASE OF MEDICAL RECORD PATIENTS
}

\author{
Rolly Intan ${ }^{1}$, Oviliani Yenty Yuliana, Andreas Handojo \\ Informatics Engineering Department, Faculty of Technology Industry, Petra Christian University \\ Jl. Siwalankerto 121 - 131, Surabaya 60236, Indonesia \\ Phone: 62-31-8494830, Fax: 62-31-8436418 \\ E-mail: rintan@petra.ac.id ${ }^{1}$
}

\begin{abstract}
Mining association rules is one of the important tasks in the process of data mining application. In general, the input as used in the process of generating rules is taken from a certain data table by which all the corresponding values of every domain data have correlations one to each others as given in the table. A problem arises when we need to generate the rules expressing the relationship between two or more domains that belong to several different tables in a normalized database. To overcome the problem, before generating rules it is necessary to join the participant tables into a general table by a process called Denormalization Process. This paper shows a process of generating Multidimensional Fuzzy Association Rules mining from a normalized database of medical record patients. The process consists of two subprocesses, namely sub-process of join tables (Denormalization Process) and sub-process of generating fuzzy rules. In general, the process of generating the fuzzy rules has been discussed in our previous papers $[1,2,3$, 4]. In addition to the process of generating fuzzy rules, this paper proposes a correlation measure of the rules as an additional consideration for evaluating interestingness of provided rules.
\end{abstract}

Keywords: denormalization process, multidimensional association rules, inter-dimension association rules, data mining, fuzzy sets.

\section{INTRODUCTION}

Association rule mining finds interesting association or correlation relationship among a large data set of items $[5,6,7]$. The discovery of interesting association rules can help in decision making process.

Association rule mining that implies a single predicate is referred as a single dimensional or intradimension association rule since it contains a single distinct predicate with multiple occurrences (the predicate occurs more than once within the rule). The terminology of single dimensional or intradimension association rule is used in multidimensional database by assuming each distinct predicate in the rule as a dimension.

Here, the method of market basket analysis can be extended and used for analyzing any relational database system. For instance, database of medical track record patients is analyzed for finding association (correlation) among diseases taken from the data of complicated several diseases suffered by patients in a certain time. For example, it might be discovered a Boolean association rule "Bronchitis $\Rightarrow$ Lung Cancer" representing relation between "Bronchitis" and "Lung Cancer" which can also be written as a single dimensional association rule as follows [6]:

Rule-1

$\operatorname{disease}(X$, "Bronchitis" $) \Rightarrow \operatorname{disease}(X$, "Lung Cancer" $)$, where Disease is a given predicate and $X$ is a variable representing patient who have a kind of disease (e.g. "Bronchitis" and "Lung Cancer"). In general, "Lung Cancer" and "Bronchitis" are two different data that are taken from a certain database attribute, called items. In general, Apriori [5, 6] is used an influential algorithm for mining frequent itemsets for generating Boolean (single dimensional) association rules.

Additional relational information regarding the identity of patients, such as age, occupation, sex, address, blood type, etc., may also have a correlation to the illness of patients. Considering each database attribute as a predicate, it can therefore be interesting to mine association rules containing multiple predicates, such as:

\section{Rule-2:}

$\operatorname{age}(X, " 60 ") \wedge \operatorname{smoking}(X$, "yes" $) \Rightarrow$ disease ( $X$, "Lung Cancer"),

where there are three predicates, namely age, smoking and disease. Association rules that involve two or more dimensions or predicates can be referred to as multidimensional association rules.

To provide a more meaningful association rule, it is necessary to utilize fuzzy sets over a given database attribute called fuzzy association rule as discussed in [1]. Formally, given a crisp domain attribute $D$, any arbitrary fuzzy set (say, fuzzy set $A$ ) is defined by a membership function of the form $[8,9]$ : 


$$
A: D \rightarrow[0,1] .
$$

A fuzzy set may be represented by a meaningful fuzzy label. For example, "young", "mid age" and "old" are fuzzy sets over age that is defined on the interval $[0,100]$ as arbitrarily given by:

young $(x)= \begin{cases}1 & \text { when } x \leq 20 \\ (35-x) / 15 & \text { when } 20<x<35 \\ 0 & \text { when } x \geq 35\end{cases}$

mid_age $(x)= \begin{cases}(x-35) / 15 & \text { when } 35<x \leq 50 \\ (65-x) / 15 & \text { when } 50<x<65 \\ 0 & \text { when } x \geq 65\end{cases}$

old $(x)= \begin{cases}(x-65) / 15 & \text { when } 65<x<80 \\ 1 & \text { when } x \geq 80\end{cases}$

Using the previous definition of fuzzy sets on age, an example of multidimensional fuzzy association rule relation among the predicates age, smoking and disease may then be represented by:

\section{$\underline{\text { Rule-3 }}$}

age $(X$, "young" $) \wedge$ smoking $(X$, "yes" $) \Rightarrow$ disease ( $X$,"bronchitis"),

To generate multidimensional association rules implying fuzzy value such as given by the above example (Rule-3) from a normalized database that consists of several tables, this paper discussed two sequential processes as shown in Figure 1.

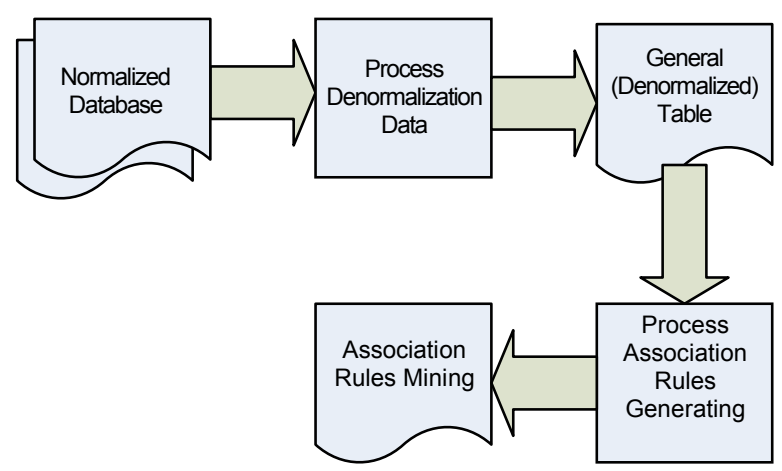

Figure 1. Process of Mining Association Rules from a Normalized Database

First is the process of joining tables known as Denormalization Process of Database. Second is the process of generating (mining) fuzzy association rules. The process of joining tables (denormalization process) can be provided based on the relation of tables as presented in Entity Relationship Diagram (ERD) of the relational database. For two tables that have no direct relation in ERD, they can still be joined by others transition tables (in ERD) using the transitive process. Other solution is that we can define or create a relation function or a relation table that correspond two distinct domains of the tables. Here, a metadata can be constructed as a data dictionary to express the relationship of tables. Result of denormalization data process is a single general table. The table is used as a source data for the process of generating rules. Further detail explanation of denormalization data process will be given in Section 3. The process generating fuzzy rules has been proposed in the paper $[6,8]$ that introduced some formulations for calculating support and confidence factors. This paper recalls the concepts proposed in $[6,8]$ and introduces an alternating formula to calculate correlation factor of a rule as also usually used in evaluating interestingness of a rule.

The structure of the paper is the following. In Section 2, basic definition and formulation of some measures, support correlation and confidence rule as used for determining interestingness of association rules are briefly recalled. Section 3 as a main contribution of this paper is devoted to propose data preparation for the further process of generation rules. Here, we will discuss a process of join table from a normalized database. Section 4 discusses a concept for mining multidimensional fuzzy association rules. Section 5 demonstrated the concept in an illustrative example. Finally a conclusion is given in Section 6.

\section{ASSOCIATION RULES, SUPPORT AND CON- FIDENCE}

Association rules are kind of patterns representing correlation of attribute-value (items) in a given set of data provided by a process of data mining system. Generally, association rule is a conditional statement (such kind of if-then rule). More formally [8], association rules are the form $A \Rightarrow B$, that is, $a_{1} \wedge \cdots \wedge a_{m} \Rightarrow b_{1} \wedge \cdots \wedge b_{n}$, where $a_{i}$ (for $i \in$ $\{1, \ldots, m\})$ and $b_{j}($ for $j \in\{1, \ldots, n\})$ are two items (attribute-value). The association rule $A \Rightarrow B$ is interpreted as "database tuples that satisfy the conditions in A are also likely to satisfy the conditions in $B^{\prime \prime} . A=\left\{a_{1}, \cdots, a_{m}\right\}$ and $\mathrm{B}=\left\{b_{1}, \cdots, b_{n}\right\}$ are two distinct itemsets. Performance or interestingness of an association rule is generally determined by three factors, namely confidence, support and correlation factors. Confidence is a measure of certainty to assess the validity of the rule. Given a set of relevant data tuples (or transactions in a transaction database) the confidence of " $A \Rightarrow B$ " is defined by:

$$
\text { confidence }(A \Rightarrow B)=\frac{\# \text { tuples }(A \text { and } B)}{\# \text { tuples }(A)} \text {, }
$$


where \#tuples $(A$ and $B)$ means the number of tuples containing $A$ and $B$.

For example, a confidence $80 \%$ for the Association Rule (for example Rule-1) means that $80 \%$ of all patients who infected bronchitis are likely to also have lung cancer. The support of an association rule refers to the percentage of relevant data tuples (or transactions) for which the pattern of the rule is true. For the association rule " $A \Rightarrow B$ " where $A$ and $B$ are the sets of items, support of the rule can be defined by

$$
\begin{aligned}
\operatorname{support}(A \Rightarrow B) & =\operatorname{support}(A \cup B) \\
& =\frac{\# \text { tuples }(A \text { and } B)}{\# \text { tuples }(\text { all_data })},
\end{aligned}
$$

where \#tuples(all_data) is the number of all tuples in the relevant data tuples (or transactions).

For example, a support $30 \%$ for the Association Rule (e.g., Rule-1) means that $30 \%$ of all patients in the all data medical records are infected both bronchitis and lung cancer. From (3), it can be followed $\operatorname{support}(A \Rightarrow B)=\operatorname{support}(B \Rightarrow A)$. Also, (2) can be calculated by

$$
\operatorname{confidence}(A \Rightarrow B)=\frac{\operatorname{support}(A \cup B)}{\operatorname{support}(A)},
$$

Correlation factor is another kind of measures to evaluate correlation between A and B. Simply, correlation factor can be calculated by:

$$
\begin{aligned}
\operatorname{correlation}(A \Rightarrow B) & =\operatorname{correlation}(B \Rightarrow A) \\
= & \frac{\operatorname{support}(A \cup B)}{\operatorname{support}(A) \times \operatorname{support}(B)},
\end{aligned}
$$

Itemset $\mathrm{A}$ and $\mathrm{B}$ are dependent (positively correlated) iff correlation $(A \Rightarrow B)>1$. If the correlation is equal to 1, then $A$ and $B$ are independent (no correlation). Otherwise, A and B are negatively correlated if the resulting value of correlation is less than 1 .

A data mining system has the potential to generate a huge number of rules in which not all of the rules are interesting. Here, there are several objective measures of rule interestingness. Three of them are measure of rule support, measure of rule confidence and measure of correlation. In general, each interestingness measure is associated with a threshold, which may be controlled by the user. For example, rules that do not satisfy a confidence threshold (minimum confidence) of, say $50 \%$ can be considered uninteresting. Rules below the threshold (minimum support as well as minimum confidence) likely reflect noise, exceptions, or minority cases and are probably of less value. We may only consider all rules that have positive correlation between its itemsets.

\section{DENORMALIZATION DATA}

In general, the process of mining data for discovering association rules has to be started from a single table (relation) as a source of data representing relation among item data. Formally, a relational data table [10] $R$ consists of a set of tuples, where $t_{i}$ represents the $i$-th tuple and if there are $n$ domain attributes $D$, then $t_{i}=\left(d_{i 1}, d_{i 2}, \cdots, d_{i n}\right)$. Here, $d_{i j}$ is an atomic value of tuple $t_{i}$ with the restriction to the domain $D_{j}$, where $d_{i j} \in D_{j}$. Formally, a relational data table $R$ is defined as a subset of the set of cross product $D_{1} \times D_{2} \times \cdots \times D_{n}$, where $D=\left\{D_{1}, D_{2}, \cdots, D_{n}\right\}$. Tuple $t$ (with respect to $R$ ) is an element of $R$. In general, $R$ can be shown in Table 1 .

\section{Table 1. A Schema of Relational Data Table}

\begin{tabular}{ccccc}
\hline Tuples & $\boldsymbol{D}_{\boldsymbol{1}}$ & $\boldsymbol{D}_{\mathbf{2}}$ & $\ldots$ & $\boldsymbol{D}_{\boldsymbol{n}}$ \\
\hline$t_{1}$ & $d_{11}$ & $d_{12}$ & $\ldots$ & $d_{1 n}$ \\
$t_{2}$ & $d_{21}$ & $d_{22}$ & $\ldots$ & $d_{2 n}$ \\
$\cdot$ & $\cdot$ & $\cdot$ & & $\cdot$ \\
$\cdot$ & $\cdot$ & $\cdot$ & & $\cdot$ \\
$\cdot$ & $\cdot$ & $\cdot$ & & $\cdot$ \\
$t_{r}$ & $\dot{d}_{r 1}$ & $\dot{d}_{r 2}$ & $\ldots$ & $\dot{d}_{m}$ \\
\hline
\end{tabular}

A normalized database is assumed as a result of a process of normalization data in a certain context of data. The database may consist of several relational data tables in which they have relation one to each others. Their relation may be represented by Entities Relationship Diagram (ERD). Hence, suppose we need to process some domains (columns) data that are parts of different relational data tables, all of the involved tables have to be combined (joined) together providing a general data table. Since the process of joining tables is an opposite process of normalization data by which the result of general data table is not a normalized table, simply the process is called Denormalization of Data (DD), and the general table is then called denormalized table. In the process of $\mathrm{DD}$, it is not necessary that all domains (fields) of the all combined tables have to be included in the targeting table. Instead, the targeting denormalized table only consists of interesting domains data that are needed in the process of mining rules. The process of DD can be performed based on two kinds of data relation as follows.

\section{The Process of Denormalized Data Based on Metadata of the Normalized database}

Information of relational tables can be stored in a metadata. Simply, a metadata can be represented by a table. Metadata can be constructed using the infor- 
mation of relational data as given in Entity Relationship Diagram (ERD). For instance, given a symbolic ERD physical design is arbitrarily shown in Figure 2. From the example, it is clearly seen that there are four tables: A, B, C and D. Here, all tables are assumed to be independent for they have their own primary keys. Cardinality of relationship between Table A and C is supposed to be one to many relationships. It is similar to relationship between Table $\mathrm{A}$ and $\mathrm{B}$ as well as Table B and C.

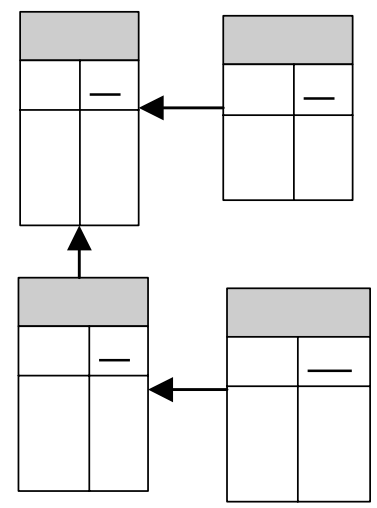

Figure 2. Example of ERD Physical Design

Table A consists of four domains/fields, D1, D2, D3 and D4; Table B also consists of four domains/fields, D1, D5, D6 and D7; Table C consists of three domains/fields, D1, D8 and D9; Table D consists of four domains/fields, D10, D11, D12 and D5. Therefore, there are totally 12 domains data as given by $\mathrm{D}=\{\mathrm{D} 1, \mathrm{D} 2, \mathrm{D} 3, \ldots, \mathrm{D} 11, \mathrm{D} 12\}$. Relationship between A and B is conducted by domain D1. Table A and $\mathrm{C}$ is also connected by domain D1. On the other hand, relationship between $\mathrm{B}$ and D is conducted by D5. Relation among A, B, C and $\mathrm{D}$ can be also represented by graph as shown in Figure 3.

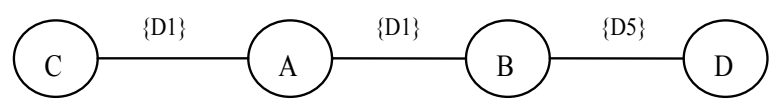

\section{Figure 3.Graph Relation of Entities}

Metadata expressing relation among four tables as given in the example can be simply seen in Table 2.

Table 2. Example of Metadata

\begin{tabular}{ccc}
\hline Table-1 & Table-2 & Relationship \\
\hline Table A & Table B & $\{\mathrm{D} 1\}$ \\
Table A & Table C & $\{\mathrm{D} 1\}$ \\
Table B & Table D & $\{\mathrm{D} 5\}$ \\
\hline
\end{tabular}

Through the metadata as given in the example, we may construct six possibilities of denormalized table as shown in Table 3.

Table 3. Possibilities of Denormalized Tables

\begin{tabular}{cl}
\hline No. & De-normalized Table \\
\hline 1 & $\mathrm{CA}(\mathrm{D} 1, \mathrm{D} 2, \mathrm{D} 3, \mathrm{D} 4, \mathrm{D} 8, \mathrm{D} 9) ; \mathrm{CA}(\mathrm{D} 1, \mathrm{D} 2, \mathrm{D} 8, \mathrm{D} 9) ;$ \\
& $\mathrm{CA}(\mathrm{D} 1, \mathrm{D} 3, \mathrm{D} 4, \mathrm{D} 9)$, etc. \\
2 & $\mathrm{CAB(D1,D2,D3,D4,D8,D9,D5,D6,D7),CAB(D1,}$ \\
& D2, D4, D9, D5,D7), etc. \\
3 & $\mathrm{CABD(D1,D2,D3,D4,D5,D6,D7,D8,D9,D10,D11,}$ \\
& D12), etc. \\
4 & $\mathrm{AB(D1,D2,D3,D4,D5,D6,D7),} \mathrm{etc.}$ \\
5 & $\mathrm{ABD(D1,D2,D3,D4,D5,D6,D7,D10,D11,D12),} \mathrm{etc.}$ \\
6 & $\mathrm{BD(D5,D6,D7,D10,D11,D12),} \mathrm{etc.}$ \\
\hline
\end{tabular}

CA(D1,D2,D3,D4,D8,D9) means that Table A and $\mathrm{C}$ are joined together, and all their domains are participated as a result of joining process. It is not necessary to take all domains from all joined tables to be included in the result, e.g. CA(D1,D2,D8,D9), $\mathrm{CAB}(\mathrm{D} 1, \mathrm{D} 2, \mathrm{D} 4, \mathrm{D} 9, \mathrm{D} 5, \mathrm{D} 7)$ and so on. In this case, what domains included as a result of the process depends on what domains are needed in the process of mining rules. For D1 and D5 are connecting domains, they are mandatory included in the result of process.

\section{The process of Denormalized Data is Based on a Data Relationship Function Defined by User.}

It is possible for user to define a mathematical function (or table) relation for connecting two or more domains from two different tables in order to perform a relationship between their entities. Generally, the data relationship function performs a mapping process from one or more domains from an entity to one or more domains from its partner entity. Hence, considering the number of domains involved in the process of mapping, it can be verified that there are four possibility relations of mapping. Let $A=\left(A_{1}, A_{2}, \cdots, A_{n}\right)$ and $B=\left(B_{1}, B_{2}, \cdots, B_{m}\right)$ be two different entities (tables). Four possibilities of function $f$ performing a mapping process are given by:

$\circ$ One to one relationship

$$
f: A_{i} \rightarrow B_{k}
$$

○ One to many relationship

$$
\begin{aligned}
& f: A_{i} \rightarrow B_{p 1} \times B_{p 2} \times \cdots \times B_{p k} \\
& \text { - Many to one rellabibe } \mathbf{A}^{p} \\
& f: A_{m 1} \times A_{m 2} \times \cdots \times A_{m k} \rightarrow B_{k} \\
& \begin{aligned}
\circ & \text { Many to many relationship } \\
& f: A_{m 1} \times A_{m} \mathbf{P} \times A_{m k} \mathbf{D} \mathbf{1}_{p 1} \times B_{p 2} \times \cdots \times B_{p k}
\end{aligned}
\end{aligned}
$$


Obviously, there is no any requirement considering type and size of data between domains in $\mathrm{A}$ and domains in B. All connections, types and sizes of data are absolutely dependent on function $f$. Construction of de-normalizations data is then performed based on the defined function.

\section{MULTIDIMENSIONAL ASSOCIATION RULES}

As explained in Section 1, association rules that involve two or more dimensions or predicates can be referred to as multidimensional association rules. Multidimensional rules with no repeated predicates are called interdimension association rules (e.g. Rule2) [11]. On the other hand, multidimensional association rules with repeated predicates, which contain multiple occurrences of some predicates, are called hybrid-dimension association rules. The rules may be also considered as combination (hybridization) between intradimension association rules and interdimension association rules. An example of such a rule is the following, where the predicate buys is repeated:

Rule-4:

age $(X$, "young" $) \wedge$ disease $(X$, "bronchitis" $) \Rightarrow$ disease ( $X$, "lung cancer").

Here, we may firstly be interested in mining multidimensional association rules with no repeated predicates or interdimension association rules. Hybriddimension association rules as an extended concept of multidimensional association rules will be discussed later in our next paper.

The interdimension association rules may be generated from a relational database or data warehouse with multiple attributes by which each attribute is associated with a predicate. To generate the multidimensional association rules, we introduce an alternative method for mining the rules by searching for the predicate sets. Conceptually, a multidimensional association rule, $A \Rightarrow B$ consists of $A$ and $B$ as two datasets, called premise and conclusion, respectively.

Formally, $A$ is a dataset consisting of several distinct data, where each data value in $A$ is taken from a distinct domain attribute in $D$ as given by

$$
A=\left\{a_{j} \mid a_{j} \in D_{j}, \text { for some } j \in \mathrm{N}_{n}\right\},
$$

where, $D_{A} \subseteq D$ is a set of domain attributes in which all data values of $A$ come from.

Similarly,

$$
B=\left\{b_{j} \mid b_{j} \in D_{j}, \text { for some } j \in \mathrm{N}_{n}\right\},
$$

where, $D_{B} \subseteq D$ is a set of domain attributes in which all data values of $B$ come from.

For example, from Rule-2, it can be found that $A=\{60$, yes $\}, B=\{$ Lung Cancer $\}, D_{A}=\{$ age, smoking $\}$ and $D_{B}=$ \{disease $\}$.

Considering $A \Rightarrow B$ is an interdimension association rule, it can be proved that $\left|D_{A}\right|=|A|$, $\left|D_{B}\right|=|B|$ and $D_{A} \cap D_{B}=\varnothing$.

Support of $A$ is then defined by:

$$
\operatorname{support}(A)=\frac{\left|\left\{t_{i} \mid d_{i j}=a_{j}, \forall a_{j} \in A\right\}\right|}{r},
$$

where $r$ is the number of records or tuples (see Table 2).

Alternatively, $r$ in (6) may be changed to $\left|Q D\left(D_{A}\right)\right|$ by assuming that records or tuples, involved in the process of mining association rules are records in which data values of a certain set of domain attributes, $D_{A}$, are not null data. Hence, (6) can be also defined by:

$$
\operatorname{support}(A)=\frac{\left|\left\{t_{i} \mid d_{i j}=a_{j}, \forall a_{j} \in A\right\}\right|}{\left|Q D\left(D_{A}\right)\right|},
$$

where $Q D\left(D_{A}\right)$, simply called qualified data of $D_{A}$, is defined as a set of record numbers $\left(t_{i}\right)$ in which all data values of domain attributes in $D_{A}$ are not null data. Formally, $Q D\left(D_{A}\right)$ is defined as follows.

$$
Q D\left(D_{A}\right)=\left\{t_{i} \mid t_{i}\left(D_{j}\right) \neq n u l l, \forall D_{j} \in D_{A}\right\} .
$$

Similarly,

$$
\operatorname{support}(B)=\frac{\left|\left\{t_{i} \mid d_{i j}=b_{j}, \forall b_{j} \in B\right\}\right|}{\left|Q D\left(D_{B}\right)\right|} .
$$

As defined in (3), support $(A \Rightarrow B)$ is given by

$$
\begin{aligned}
\operatorname{support}(A \Rightarrow B) & =\operatorname{support}(A \cup B) \\
& =\frac{\left|\left\{t_{i} \mid d_{i j}=c_{j}, \forall c_{j} \in A \cup B\right\}\right|}{\left|Q D\left(D_{A} \cup D_{B}\right)\right|}
\end{aligned}
$$

confidence $(A \Rightarrow B)$ as a measure of certainty to assess the validity of $A \Rightarrow B$ is calculated by

$\operatorname{confidence}(A \Rightarrow B)=\frac{\left|\left\{t_{i} \mid d_{i j}=c_{j}, \forall c_{j} \in A \cup B\right\}\right|}{\left|\left\{t_{i} \mid d_{i j}=a_{j}, \forall a_{j} \in A\right\}\right|}$

If support $(A)$ is calculated by ( 6 ) and denominator of (10) is changed to $r$, clearly, (10) can be proved having relation as given by (4).

$A$ and $B$ in the previous discussion are datasets in which each element of $A$ and $B$ is an atomic crisp value. To provide a generalized multidimensional association rules, instead of an atomic crisp value, we 
may consider each element of the datasets to be a dataset of a certain domain attribute. Hence, $A$ and $B$ are sets of set of data values. For example, the rule may be represented by

Rule-5:

$\operatorname{age}(X, " 20 \ldots 60 ") \wedge \operatorname{smoking}(X$, ,yes" $) \Rightarrow$ disease ( $X$, "bronchitis, lung cancer"),

where $A=\{\{20 \ldots 29\},\{$ yes $\}\}$ and $\mathrm{B}=\{\{$ bronchitis, lung cancer $\}\}$.

Simply, let $A$ be a generalized dataset. Formally, $A$ is given by $A=\left\{A_{j} \mid A_{j} \subseteq D_{j}\right.$, for some $\left.j \in \mathrm{N}_{n}\right\}$.

Corresponding to (7), support of $A$ is then defined by:

$$
\operatorname{support}(A)=\frac{\left|\left\{t_{i} \mid d_{i j} \subseteq A_{j}, \forall A_{j} \in A\right\}\right|}{\left|Q D\left(D_{A}\right)\right|} .
$$

Similar to (10),

$\operatorname{support}(A \Rightarrow B)=\operatorname{support}(A \cup B)$

$$
=\frac{\left|\left\{t_{i} \mid d_{i j} \subseteq C_{j}, \forall C_{j} \in A \cup B\right\}\right|}{\left|Q D\left(D_{A} \cup D_{B}\right)\right|}
$$

Finally, confidence $(A \Rightarrow B)$ is defined by

$\operatorname{confidence}(A \Rightarrow B)=\frac{\left|\left\{t_{i} \mid d_{i j} \subseteq C_{j}, \forall C_{j} \in A \cup B\right\}\right|}{\left|\left\{t_{i} \mid d_{i j} \subseteq A_{j}, \forall A_{j} \in A\right\}\right|}$

To provide a more generalized multidimensional association rules, we may consider $A$ and $B$ as sets of fuzzy labels. Simply, $A$ and $B$ are called fuzzy datasets. Rule-3 is an example of such rules, where $A=\{$ young, yes $\}$ and $\mathrm{B}=\{$ bronchitis $\}$. A fuzzy dataset is a set of fuzzy data consisting of several distinct fuzzy labels, where each fuzzy label is represented by a fuzzy set on a certain domain attribute. Let $A$ be a fuzzy dataset. Formally, $A$ is given by

$$
A=\left\{A_{j} \mid A_{j} \in \mathrm{F}\left(D_{j}\right) \text {, for some } j \in \mathrm{N}_{n}\right\},
$$

where $\mathrm{F}\left(D_{j}\right)$ is a fuzzy power set of $D_{j}$, or in other words, $A_{j}$ is a fuzzy set on $D_{j}$. Corresponding to (7), support of $A$ is then defined by:

$$
\operatorname{support}(A)=\frac{\sum_{i=1}^{r} \inf _{A_{j} \in A}\left\{\mu_{A_{j}}\left(d_{i j}\right)\right\}}{\left|Q D\left(D_{A}\right)\right|} .
$$

Similar to (10),

$$
\begin{aligned}
\operatorname{support}(A \Rightarrow B)= & \operatorname{support}(A \cup B) \\
& =\frac{\sum_{i=1}^{r} \inf _{C_{j} \in A \cup B}\left\{\mu_{C_{j}}\left(d_{i j}\right)\right\}}{\left|Q D\left(D_{A} \cup D_{B}\right)\right|}
\end{aligned}
$$

Confidence $(A \Rightarrow B)$ is defined by

$$
\operatorname{confidence}(A \Rightarrow B)=\frac{\sum_{i=1}^{r} \inf _{C_{j} \in A \cup B}\left\{\mu_{C_{j}}\left(d_{i j}\right)\right\}}{\sum_{i=1}^{r} \inf _{A_{j} \in A}\left\{\mu_{A_{j}}\left(d_{i j}\right)\right\}}
$$

Finally, correlation $(A \Rightarrow B)$ is defined by

$$
\operatorname{correlation}(A \Rightarrow B)=\frac{\sum_{i=1}^{r} \inf _{C_{j} \in A \cup B}\left\{\mu_{C_{j}}\left(d_{i j}\right)\right\}}{\sum_{i=1}^{r} \inf _{A_{j} \in A}\left\{\mu_{A_{j}}\left(d_{i j}\right)\right\} \times \inf _{B_{k} \in B}\left\{\mu_{B_{k}}\left(d_{i k}\right)\right\}}
$$

Similarly, if denominators of (15) and (16) are changed to $r$ (the number of tuples), (17) can be proved also having relation as given by (4). Here, we may consider and prove that (16) and (17) are generalization of (13) and (14), respectively. On the other hand, (13) and (14) are generalization of (10) and (11).

\section{ILLUSTRATIVE EXAMPLE}

An illustrative example is given to understand well the concept of the proposed method and how to calculate support, confidence and correlation of the multidimensional fuzzy association rule is performed. The process is started from a given a simple medical records of patients as shown in Table 4.

\section{Table 4. Medical Records of Patients}

\begin{tabular}{ccccl}
\hline Tuples & Ages & Smoking & \multicolumn{3}{c}{ Blood Type Diseases } \\
\hline$t_{1}$ & 20 & yes & A & bronchitis \\
$t_{2}$ & 25 & yes & A & bronchitis \\
$t_{3}$ & 22 & yes & B & bronchitis \\
$t_{4}$ & 27 & No & O & diarrhea \\
$t_{5}$ & 30 & No & O & diarrhea \\
$t_{6}$ & 45 & yes & AB & lung cancer \\
$t_{7}$ & 40 & yes & O & lung cancer \\
$t_{8}$ & 50 & No & O & diabetes \\
$t_{9}$ & 60 & yes & B & bronchitis \\
$t_{10}$ & 60 & yes & A & lung cancer \\
$t_{11}$ & Null & No & AB & diarrhea \\
\hline
\end{tabular}

Based on Table 4, support and confidence of Rule-2 are calculated using (10) and (11), respectively. Related to the conceptual form of the rule $A \Rightarrow B$, it can be followed that $A=\{60$, yes $\}$ and $B=\{$ lung cancer $\}$. 


$$
\operatorname{support}(\text { Rule }-2)=\frac{\left|\left\{t_{10}\right\}\right|}{\left|\left\{t_{1}, \ldots, t_{10}\right\}\right|}=0.1,
$$

where $Q D\left(D_{A} \cup D_{B}\right)=\left\{t_{1}, \ldots, t_{10}\right\} . t_{11}$ is not included in $Q D\left(D_{A} \cup D_{B}\right)$, because it has a null value in Ages. Confidence of Rule- 2 is given by

$$
\text { confidence }(\text { Rule }-2)=\frac{\left|\left\{t_{10}\right\}\right|}{\left|\left\{t_{10}, t_{9}\right\}\right|}=0.5 \text {. }
$$

Correlation of Rule- 2 is given by

$$
\begin{aligned}
\operatorname{correlation}(\text { Rule }-2) & =\frac{\operatorname{support}(\text { Rule }-2)}{\operatorname{support}(\{60, \text { yes }\}) \times \operatorname{support}(\{\text { bronchitis }\})} \\
& =\frac{0.1}{0.2 \times 0.4}=1.25
\end{aligned}
$$

Support, confidence and correlation of Rule-5 are calculated using (13) and (14) as follows.

$$
\begin{gathered}
\operatorname{support}\left(\text { Rule - 5) }=\frac{\left|\left\{t_{1}, t_{2}, t_{3}, t_{6}, t_{7}, t_{9}, t_{10}\right\}\right|}{\left|\left\{t_{1}, \ldots, t_{10}\right\}\right|}=0.7,\right. \\
\text { confidence(Rule - 5) }=\frac{\left|\left\{t_{1}, t_{2}, t_{3}, t_{6}, t_{7}, t_{9}, t_{10}\right\}\right|}{\left|\left\{t_{1}, t_{2}, t_{3}, t_{6}, t_{7}, t_{9}, t_{10}\right\}\right|}=1 . \\
\text { correlation(Rule-5) }=\frac{0.7}{0.7 \times 0.7}=1.43
\end{gathered}
$$

Rule- 3 is a fuzzy rule, where $A=\{$ young, yes $\}$ and $\mathrm{B}=\{$ bronchitis $\}$. Young $(y g)$ is a fuzzy labels represented by a fuzzy sets as given in Section 1. Support of Rule-3 can be calculated by (16) as shown in the following table.

Table 5. Calculation of Fuzzy Values

\begin{tabular}{ccccc}
\hline Tuples & $\mu_{y g}($ ages $)$ & $\mu_{y s}($ smk $)$ & $\mu_{b r}($ dis $)$ & $\begin{array}{l}\text { Min } \\
\end{array}$ \\
$\boldsymbol{\alpha}$ & $\boldsymbol{\beta}$ & $\boldsymbol{\gamma}$ & $(\boldsymbol{\alpha}, \boldsymbol{\beta}, \boldsymbol{\gamma})$ \\
\hline$t_{1}$ & 1 & 1 & 1 & 1 \\
$t_{2}$ & 0.66 & 1 & 1 & 0.66 \\
$t_{3}$ & 0.87 & 1 & 1 & 0.87 \\
$t_{4}$ & 0.53 & 0 & 0 & 0 \\
$t_{5}$ & 0.33 & 0 & 0 & 0 \\
$t_{6}$ & 0 & 1 & 0 & 0 \\
$t_{7}$ & 0 & 1 & 0 & 0 \\
$t_{8}$ & 0 & 0 & 0 & 0 \\
$t_{9}$ & 0 & 1 & 1 & 0 \\
$t_{10}$ & 0 & 1 & 0 & 0 \\
$t_{11}$ & $n u l l$ & 0 & 0 & 0 \\
$\Sigma$ & 3.4 & 7 & 4 & 2.53 \\
\hline
\end{tabular}

Therefore,

$$
\operatorname{support}(\text { Rule }-3)=\frac{2.53}{\left|\left\{t_{1}, \ldots, t_{10}\right\}\right|}=0.253
$$

On the other hand, confidence and correlation of Rule- 3 are given by

$$
\begin{gathered}
\text { confidence }(\text { Rule }-3)=\frac{2.53}{2.53}=1 . \\
\text { correlation }(\text { Rule- } 3)=\frac{0.253}{0.253 \times 0.4}=2.5
\end{gathered}
$$

Positive results of correlations, Rule-1, Rule- 5 and Rule-3 show that their conclusion and condition sides are not independent.

\section{CONCLUSION}

The paper firstly discussed a method of how to provide a denormalized table from a normalized database. Then, a concept of generating multidimensional fuzzy association rules was introduced in the context of mining association rules from medical records of patients. In general, multidimensional association rules consist of two types of rules, namely interdimension association rules and hybriddimension association rules. In this paper, we still restricted our proposed extended method to generate interdimension association rules. Three sets of equations were introduced to calculate support, confidence and correlation of three different kinds of generalized rules. In our next paper, we will discuss and propose a method to generate hybrid-dimension association rules by assuming that hybrid-dimension association rules is a hybridization between intradimension and interdimension association rules.

\section{Acknowledgement}

This work has been supported by the research grant of The Higher Education Directorate of Indonesia (Penelitian Hibah Bersaing) in the year of 2007.

\section{REFERENCES}

1. Intan, Rolly, A Proposal of Fuzzy Multidimensional Association Rules, Jurnal Informatika, Vol. 7 No. 2 (Terakreditasi SK DIKTI No. 56/DIKTI/ Kep/2005), November 2006.

2. Intan, Rolly, A Proposal of an Algorithm for Generating Fuzzy Association Rule Mining in Market Basket Analysis, Proceeding of CIRAS (IEEE). Singapore, 2005.

3. Intan, Rolly, An Algorithm for Generating Single Dimensional Association Rules, Jurnal Informatika, Vol. 7 No. 1 (Terakreditasi SK DIKTI No. 56/DIKTI/Kep/2005), May 2006.

4. Intan, Rolly, Generating Multi Dimensional Association Rules Implying Fuzzy Valuse, The 
International Multi-Conference of Engineers and Computer Scientist, Hong Kong, 2006.

5. Agrawal, Tomasz Imielinski, Arun N. Swami, Mining Association Rules between Sets of Items in Large Database, Proccedings of ACM SIGMOD International Conference Management of Data, ACM Press, 1993, pp. 207-216.

6. Han, Jiawei, Micheline Kamber, Data Mining: Concepts and Techniques, The Morgan Kaufmann Series, 2001.

7. Intan, Rolly, A Proposal of an Algorithm for Generating Fuzzy Association Rule Mining in Market Basket Analysis, Proceedings of The $3^{\text {rd }}$ International Conference on Computational Intelligence, Robotics and Autonomous System, 2005.
8. Klir, George, Bo Yuan, Fuzzy Sets and Fuzzy Logic: Theory and Applications, New Jersey: Prentice Hall, 1995.

9. Zadeh, Lotfi A., Fuzzy Sets and systems, International Journal of General Systems, Vol. 17, 1990, pp. 129-138.

10. Codd, Edgar F., Communication of the ACM 13 (6), 1970, pp. 377-387.

11. Agrawal, Rakesh, Ramakrishnan Srikant, Fast Algorithms for Mining Association Rules in Large Databases, Proccedings of $20^{\text {th }}$ International Conference Very Large Databases, Morgan Kaufman, 1994, pp. 487-499. 